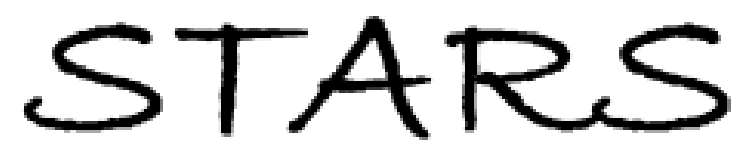

University of Central Florida

STARS

Faculty Bibliography 2000s

Faculty Bibliography

$1-1-2006$

\title{
In situ experimental study of a near-field lens at visible frequencies
}

\author{
G. Webb-Wood \\ University of Central Florida
}

\author{
A. Ghoshal \\ University of Central Florida \\ P. G. Kik \\ University of Central Florida
}

Find similar works at: https://stars.library.ucf.edu/facultybib2000

University of Central Florida Libraries http://library.ucf.edu

This Article is brought to you for free and open access by the Faculty Bibliography at STARS. It has been accepted for inclusion in Faculty Bibliography 2000 s by an authorized administrator of STARS. For more information, please contactSTARS@ucf.edu.

\section{Recommended Citation}

Webb-Wood, G.; Ghoshal, A.; and Kik, P. G., "In situ experimental study of a near-field lens at visible frequencies" (2006). Faculty Bibliography 2000s. 6702.

https://stars.library.ucf.edu/facultybib2000/6702

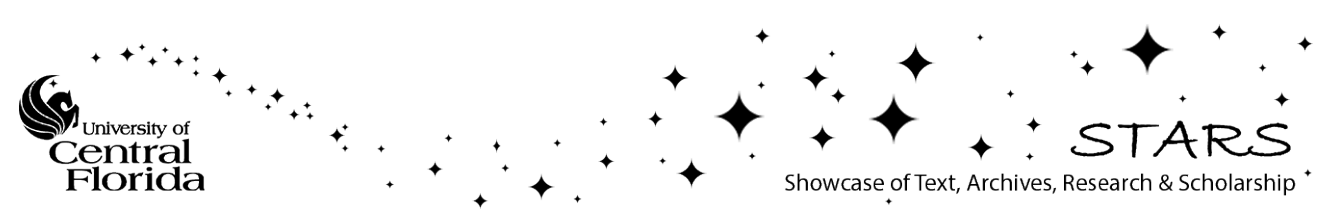




\section{In situ experimental study of a near-field lens at visible frequencies}

Cite as: Appl. Phys. Lett. 89, 193110 (2006); https://doi.org/10.1063/1.2387871

Submitted: 18 May 2006 . Accepted: 26 September 2006 . Published Online: 08 November 2006

G. Webb-Wood, A. Ghoshal, and P. G. Kik
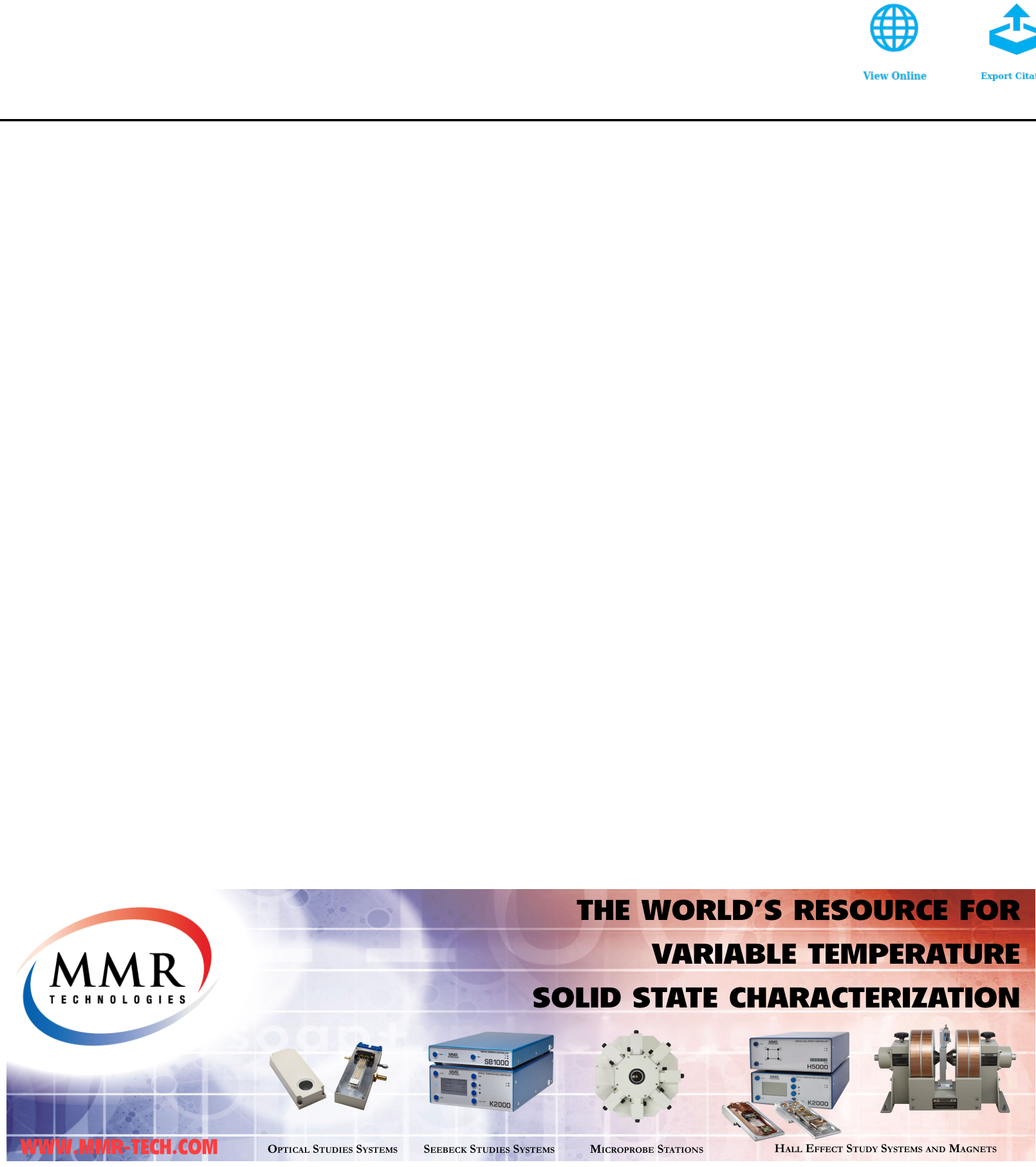


\title{
In situ experimental study of a near-field lens at visible frequencies
}

\author{
G. Webb-Wood, ${ }^{\text {a) }}$ A. Ghoshal, and P. G. Kik ${ }^{\text {b) }}$ \\ College of Optics and Photonics: CREOL \& FPCE, University of Central Florida, \\ 4000 University Boulevard, Orlando, Florida 32816
}

(Received 18 May 2006; accepted 26 September 2006; published online 8 November 2006)

\begin{abstract}
Frequency dependent near-field scanning optical microscopy (NSOM) measurements of plasmon-mediated near-field focusing using a $50 \mathrm{~nm}$ thick Au film are presented. In these studies the tip aperture of a NSOM probe acts as a localized light source, while the near-field image formed by the metal lens is detected in situ using nanoscale scatterers placed in the image plane. By scanning the relative position of object and probe, the near-field image generated by the lens is resolved. NSOM scans performed at different illumination frequencies reveal an optimum near-field image quality at frequencies close to the surface plasmon resonance frequency. (C) 2006 American Institute of Physics. [DOI: 10.1063/1.2387871]
\end{abstract}

In recent years, there has been a surge in interest in using surface plasmons (SPs) for a diverse array of applications, including improved sensors, ${ }^{1}$ enhanced optical lithography, ${ }^{2}$ and information transfer. ${ }^{3,4}$ An additional application of surface plasmons was proposed in 2000 by Pendry. ${ }^{5}$ In his seminal work, Pendry predicted that a thin metal film can act as a near-field lens capable of generating images with feature sizes smaller than the diffraction limit. For a silver film in air, this effect was theoretically predicted to occur at a frequency $\omega_{\mathrm{SP}}$, where $\varepsilon_{\mathrm{Ag}}\left(\omega_{\mathrm{SP}}\right)=-1$, coinciding with the existence of localized surface plasmons at the Ag-air interface, and it was suggested that surface plasmons played an important role in the imaging process. The role of surface plasmons in the imaging process was studied in detail in a time domain study of a silver lens, ${ }^{6}$ and the numerical results indicated that a nanoscale source placed near a thin metal film can excite localized surface plasmons on both sides of the lens, thus forming a high resolution image of the source. Theoretical studies investigated the effect of spatial dispersion, ${ }^{7}$ dielectric environment asymmetry, ${ }^{8,9}$ and surface roughness ${ }^{10}$ on the resolution of a real metallic lens. Recent experimental studies have unambiguously demonstrated improvements in the resolution of near-field lithography at visible and near-UV frequencies by using thin $(<100 \mathrm{~nm})$ Ag films. ${ }^{11-13}$ A direct study of the properties of a near-field lens by Moerland et al. ${ }^{14}$ demonstrated that the image resolution is distance dependent. In this letter, we focus on the role of surface plasmons in the imaging process by studying the frequency dependent imaging properties of a gold near-field lens.

In order to observe near-field focusing, we performed experiments based on a geometry proposed by Kik et al., ${ }^{6}$ as shown schematically in Fig. 1(a). In this approach the nearfield lens consists of a thin metal film deposited on a dielectric support layer, enabling direct experimental access to both sides of the lens structure. The tip of a near-field scanning optical microscopy (NSOM) probe is brought into contact with the dielectric spacer layer. When light is focused into the back of this metal coated tip, an approximately dipolar field distribution is generated near the aperture of the probe,

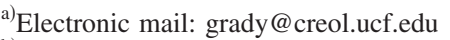

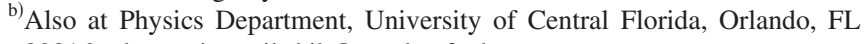
32816; electronic mail: kik@creol.ucf.edu
}

oscillating at the frequency of the incident light. ${ }^{15}$ This tightly confined field serves as the "object" in this study. Part of the light present at the aperture is transmitted through the lens and collected by a microscope objective beneath the sample. At the same time, high spatial frequency components of the tip field excite surface plasmons at the metal-dielectric interface ${ }^{16}$ generating a periodic charge distribution and corresponding field distribution on either side of the metaldielectric interface, as indicated schematically by the field lines in Fig. 1(a). A metal nanoparticle placed in the "image plane" behind the metal lens can convert part of these local fields into far-field radiation, which is also collected by the objective beneath the sample. As the relative position of the tip and metal particle is changed, the light scattered by the metal nanoparticle will experience a phase delay, given by the tip-to-particle separation and the surface plasmon wavelength, relative to the light emitted by the tip. By measuring the total collected far-field intensity as a function of the sample displacement, the interference of the tip radiation and the scattered radiation provides information about the amplitude and relative phase of the fields present in the image plane.

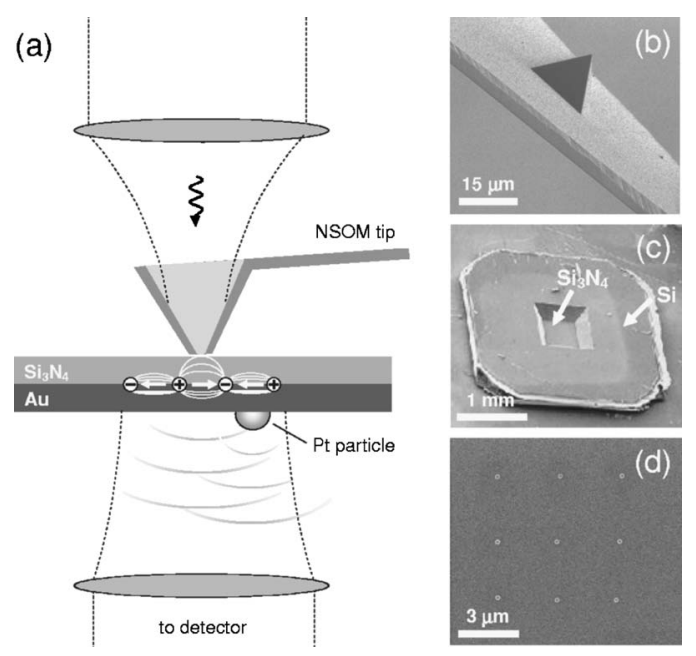

FIG. 1. (a) Schematic view of the experiment, (b) a FIB image of a nearfield optical probe, (c) a transmission electron microscopy image of a $\mathrm{Si}_{3} \mathrm{~N}_{4}$ membrane on $\mathrm{Si}$, and (d) a FIB image of the Pt dot pattern on a $50 \mathrm{~nm} \mathrm{Au}$ layer deposited on the $\mathrm{Si}_{3} \mathrm{~N}_{4}$ membrane used in the experiments. 


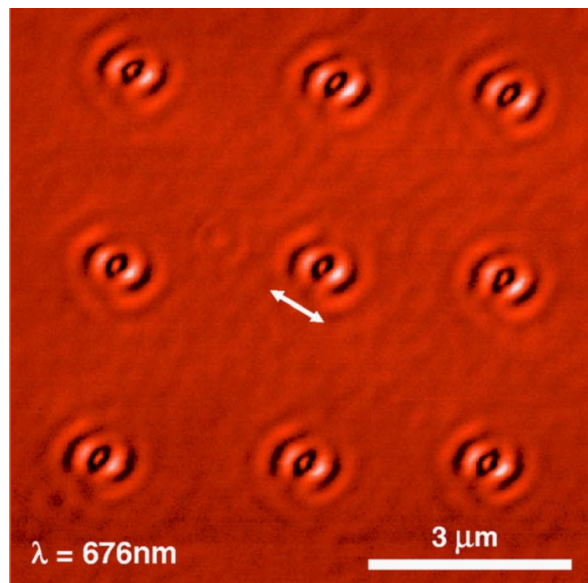

FIG. 2. (Color online) NSOM image of a suspended $\mathrm{Au}-\mathrm{Si}_{3} \mathrm{~N}_{4}$ bilayer $(50 \mathrm{~nm} / 50 \mathrm{~nm})$ containing a square array of $\mathrm{Pt}$ nanoparticles. The fringe pattern is caused by the interference of dipole radiation from the aperture of a near-field tip with surface plasmon fields scattered by the particles on the back side of the Au film. Each individual pattern represents an image of the tip field distribution generated by the metal lens and sampled by a Pt nanoparticle. The arrow shows the polarization direction of the tip field.

Figure 1(b) shows a scanning ion beam microscopy image of a near-field probe of the type used in this experiment. The NSOM tip consists of a hollow $\mathrm{SiO}_{2}$ pyramid coated with $\sim 200 \mathrm{~nm}$ thick Al layer, with a tip aperture $<100 \mathrm{~nm}$. The dielectric support layer in this study consists of a freestanding $50 \mathrm{~nm}$ thick silicon nitride window ${ }^{17}$ supported by a $200 \mu \mathrm{m}$ thick Si substrate, as shown in Fig. 1(c). A $50 \mathrm{~nm}$ thick gold film was sputtered from a $99.999 \%$ Au target onto the inner surface of the $\mathrm{Si}_{3} \mathrm{~N}_{4}$ window. Step height measurements using contact mode atomic force microscopy in the area surrounding the window confirmed the film thickness and revealed a rms surface roughness of $\pm 2 \mathrm{~nm}$, or $\pm 4 \%$ of the film thickness. A square array of Pt nanoparticles with a diameter between 150 and $200 \mathrm{~nm}$ and an interparticle spacing of $3 \mu \mathrm{m}$ was deposited on the gold surface using a FEI 200 focused ion beam (FIB) system, as shown in Fig. 1(d). NSOM measurements were accomplished using a WITec alpha-SNOM in transmission mode. Linearly polarized light from a $\mathrm{Kr}$ ion laser was focused into the back of the NSOM tip, with a typical input power into the tip of $<700 \mu \mathrm{W}$. To map out the near-field image, the sample position was scanned laterally while the light from the tip and the $\mathrm{Pt}$ particle was collected using a $20 \times$ objective (numerical aperture $=0.4$ ) positioned beneath the sample stage and projected onto a multimode fiber. The total collected light intensity was recorded as a function of position using a fiber coupled avalanche photodiode. All of the measurements were taken over an area of $9 \times 9 \mu \mathrm{m}^{2}$, with 400 pixels per line and 400 lines per scan, for a collection time of $5 \mathrm{~ms} /$ pixel. NSOM images have been scaled for optimum contrast but are otherwise unprocessed.

Figure 2 shows a NSOM measurement of the gold nearfield lens as using an illumination wavelength of $676 \mathrm{~nm}$. The polarization of the light transmitted by the tip is indicated by the arrow. The scan image shows an array of oscillatory patterns that match the spacing and location of the FIB-defined Pt nanoparticles. Each of these features represents an image of the tip field distribution generated by the gold lens. It is clear that at this frequency the metal lens does not produce any near-field focusing, but rather forms an ex-
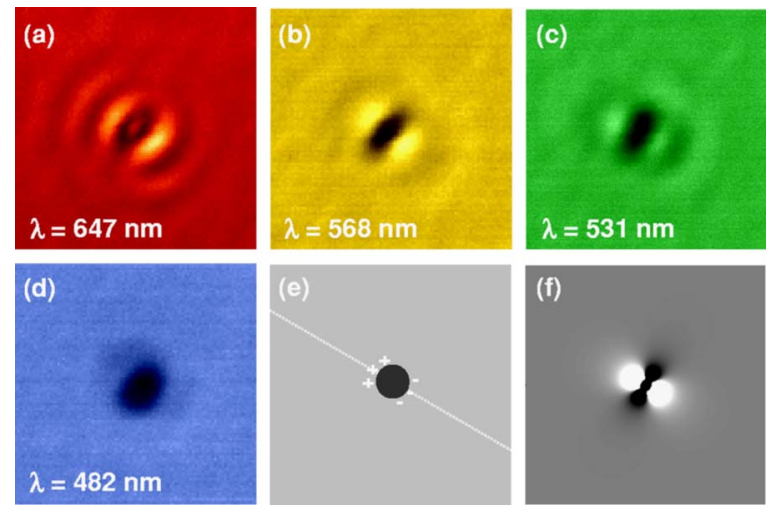

FIG. 3. (Color online) $[(a)-(d)]$ NSOM images of the central feature of the nanoparticle resolution test pattern. The observed intensity patterns represent near-field images of the tip, generated by the gold lens. The displayed area measures $2.2 \mu \mathrm{m}$ on a side. (e) Schematic representation of the charge distribution near a nanoaperture in a metal film when illuminated with linearly polarized light and (f) the corresponding field distribution close to the aperture, shown on the same scale as $[(\mathrm{a})-(\mathrm{d})]$.

tended field pattern in the image plane. This field distribution is due to the near-field excitation of surface plasmons on the gold-dielectric interface. At frequencies below $\omega_{\mathrm{SP}}$, the NSOM tip excites propagating surface plasmons with a wavelength close to the wavelength of light in the surrounding medium. The light scattered by the Pt particle interferes constructively each time the phase delay between the field near the tip and the field near the Pt particle differs by $2 \pi n$, with $n$ an integer number. The measured distance between the interference maxima of $420 \pm 5 \mathrm{~nm}$ indeed corresponds closely to the calculated plasmon wavelength of $\sim 410 \mathrm{~nm}$ for plasmons at the interface between $50 \mathrm{~nm} \mathrm{Au}$ and $50 \mathrm{~nm}$ $\mathrm{Si}_{3} \mathrm{~N}_{4}$ in air. ${ }^{18}$ It should be noted that the limited spatial extent of the fringe pattern is an intrinsic result of the collection scheme: the light projected onto the collection fiber is collected from $\mathrm{a} \sim 1 \mu \mathrm{m}$ diameter area at the focus of the collection objective. In the area between the Pt particles, small fluctuations $( \pm 3 \%)$ in the total intensity can be observed. These features are most likely caused by the surface roughness of the gold film. The measured film thickness fluctuations are of the order of $\pm 2 \mathrm{~nm}$, which at this wavelength could induce a maximum variation in linear transmission of $\pm 7 \%$ based on literature values of the dielectric function of gold. It should also be noted that the tip image detected by the central Pt particle is identical to the tip image detected by any of the outer Pt particles, suggesting that multiple scattering of SPs does not significantly affect the results.

Several NSOM scans similar to the one shown in Fig. 2 were performed using the same NSOM probe under different illumination frequencies. The results were found to be reproducible over many scans, showing that neither tip degradation nor film degradation plays a significant role in these experiments. Figure 3 shows subsequent scans made around the central particle at wavelengths of $647,568,531$, and $482 \mathrm{~nm}$. At a wavelength of $647 \mathrm{~nm}$, a pattern similar to the one in Fig. 2 is observed, with a higher periodicity due to the increased illumination frequency. When the wavelength is decreased to $568 \mathrm{~nm}$, the observed field distribution appears more localized, showing a narrow central minimum and two main maxima on either side of the minimum. This pattern closely represents the lateral field distribution around a circular aperture. For comparison, Fig. 3(f) shows a calculation 


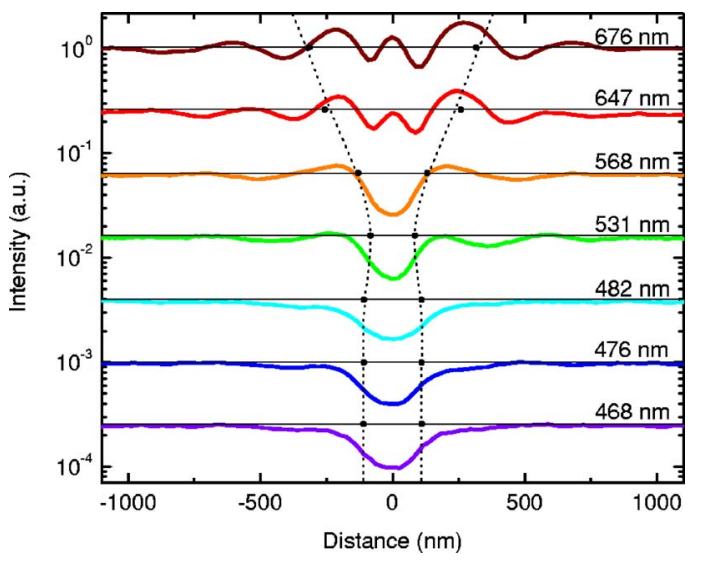

FIG. 4. (Color online) Cross sections of NSOM images, taken at different illumination wavelengths. The cross sections were taken parallel to the incident polarization. The signal amplitude has been scaled by a fixed factor for clarity. The black circles show the fitted image width for each frequency. The dashed line is a guide to the eyes.

of the field strength along the direction of polarization near a $100 \mathrm{~nm}$ diameter circular aperture illuminated with linearly polarized light. The edge of the aperture is assumed to support a surface charge density proportional to $\cos (\theta)$, as indicated in Fig 3(e), where $\theta$ represents the angle relative to the polarization of the incident light. The calculated field pattern shows a striking similarity to the observed field distribution in Fig. 3(b). Figure 3(c) shows a NSOM scan for a wavelength of $531 \mathrm{~nm}\left(\omega=3.55 \times 10^{15} \mathrm{rad} / \mathrm{s}\right)$, close to the theoretically predicted value of $\omega_{\mathrm{SP}}=3.60 \times 10^{15} \mathrm{rad} / \mathrm{s}$ of a $\mathrm{Au}-\mathrm{Si}_{3} \mathrm{~N}_{4}$ interface, revealing a localized pattern similar to the one in Fig. 3(b) with a slightly narrower central minimum. Figure 3(d) shows a NSOM scan for a wavelength of $482 \mathrm{~nm}$, well above the localized plasmon frequency. In this case, no oscillatory features are present, and a broad central minimum is observed. Since no surface plasmons exist at the $\mathrm{Au}-\mathrm{Si}_{3} \mathrm{~N}_{4}$ interface at this frequency, the image represents the direct interaction of light emitted from the tip with the $\mathrm{Pt}$ particle. This high frequency scan thus represents a reference measurement of the field distribution in the image plane in the absence of surface-plasmon-mediated focusing. The scans in Fig. 3 demonstrate that illumination at frequencies near $\omega_{\mathrm{SP}}$ results in a spatially narrowed field distribution compared to NSOM scans at frequencies where surface plasmons are not present, providing a direct experimental demonstration of SP-mediated near-field focusing.

In order to determine the frequency dependent image width, we have analyzed line cuts of the obtained NSOM data. Figure 4 shows line cuts of the intensity distributions from seven independent NSOM scans taken along the polarization direction near the central particle. The total intensity in the scans has been normalized; however, the image contrast represents that of the original scans. Assigning an image width to these intensity distributions is not a straightforward task, since the nature of the field distribution changes upon going from frequencies below the plasmon resonance to frequencies above the resonance. At low frequencies an extended sinusoidal pattern is observed, while at high frequencies an approximately Gaussian distribution is found. In order to quantitatively describe the image width, we have performed fits of the intensity data using a phenomenological model for the field distribution. The model takes into account a fixed tip field $E_{\text {tip }}$, a plasmon-mediated field contribution that contains an oscillating term, and a Gaussian amplitude distribution around the center position,

$$
I \propto\left|E_{\text {tip }}+E_{\mathrm{SP}} e^{i\left(k_{x} x+\phi\right)} e^{-2 \ln 2(x / w)^{2}}\right|^{2} .
$$

Here, $x$ is the distance from the center of the line cut, $w$ is the effective width, $E_{\mathrm{SP}}$ corresponds to the maximum field amplitude generated by the Pt particle, $k_{x}$ is the observed wave vector, and $\phi$ is a position-independent phase delay between the fields at the tip aperture and the field scattered by the Pt particle. This last parameter takes into account frequency dependent phase delays that occur in the SP excitation by the tip and the SP scattering. Such a frequency dependent phase delay was previously shown to significantly affect near-field images of Au nanoparticles. ${ }^{19}$ In the absence of an oscillatory component, $w$ reduces to the full width at half maximum of the near-field intensity distribution. The black circles in Fig. 4 show the image width obtained from the line cuts. A minimum width is observed for a wavelength of $531 \mathrm{~nm}$, providing clear evidence for surface-plasmonmediated near-field focusing. It should be noted that the observed minimum width of $165 \mathrm{~nm}$ does not represent the optimum resolution that can be obtained using a near-field lens, since the image width is limited by the finite size of the tip aperture and the particle.

In conclusion, we have demonstrated frequency dependent near-field imaging using a planar metal lens. At low frequencies, the lens was shown to produce extended field distributions due to the local excitation of propagating surface plasmons. At high frequencies, no surface-plasmonmediated effects were observed. It was shown that an optimum image resolution occurs at frequencies near the resonance frequency of localized surface plasmons, as predicted by theory.

This work was supported in part by the Partnership projects program of the Florida Photonics Center of Excellence (FPCE).

${ }^{1}$ J. Homola, Anal. Bioanal. Chem. 377, 528 (2003).

${ }^{2}$ W. Srituravanich, N. Fang, C. Sun, Q. Luo, and X. Zhang, Nano Lett. 4, 1085 (2004).

${ }^{3}$ W. L. Barnes, A. Dereux, and T. W. Ebbesen, Nature (London) 424, 824 (2003).

${ }^{4}$ S. A. Maier, P. G. Kik, H. A. Atwater, S. Meltzer, E. Harel, B. E. Koel, and A. G. Requicha, Nat. Mater. 2, 229 (2003).

5 J. B. Pendry, Phys. Rev. Lett. 85, 3966 (2000).

${ }^{6}$ P. G. Kik, S. A. Maier, and H. A. Atwater, Phys. Rev. B 69, 045418 (2004).

${ }^{7}$ I. A. Larkin and M. I. Stockman, Nano Lett. 5, 339 (2005).

${ }^{8}$ S. A. Ramakrishna, J. B. Pendry, D. Schurig, D. R. Smith, and S. Schultz, J. Mod. Opt. 49, 1747 (2002).

${ }^{9}$ R. Merlin, Appl. Phys. Lett. 84, 1290 (2004).

${ }^{10}$ R. J. Blaikie and S. J. McNab, Microelectron. Eng. 61-62, 97 (2002).

${ }^{11}$ D. O. S. Melville and R. J. Blaikie, Opt. Express 13, 2127 (2005).

${ }^{12}$ N. Fang, H. Lee, C. Sun, and X. Zhang, Science 308, 534 (2005).

${ }^{13}$ D. B. Shao and S. C. Chen, Appl. Phys. Lett. 86, 253107 (2005).

${ }^{14}$ R. J. Moerland, N. F. van Hulst, H. Gersen, and L. Kuipers, Opt. Express 13, 1604 (2005)

${ }^{15}$ R. D. Grober, T. Rutherford, and T. D. Harris, Appl. Opt. 35, 3488 (1996).

${ }^{16}$ B. Hecht, H. Bielefeldt, L. Novotny, Y. Inouye, and D. W. Pohl, Phys. Rev. Lett. 77, 1889 (1996).

${ }^{17}$ Structure Probe, Inc., West Chester, PA.

${ }^{18}$ C. A. Ward, K. Bhasin, R. J. Bell, R. W. Alexander, and I. Tyler, J. Chem. Phys. 62, 1674 (1975); 62, 4960(E) (1975).

${ }^{19}$ J. Prikulis, H. Xu, L. Gunnarsson, M. Käll, and H. Olin, J. Appl. Phys. 92, $6211(2002)$ 